\title{
Molecular detection of cattle Sarcocystis spp. in North-West Italy highlights their association with bovine eosinophilic myositis
}

\author{
Selene Rubiola ${ }^{{ }^{*}} \mathbb{D}$, Tiziana Civera ${ }^{1}$, Felice Panebianco ${ }^{1}$, Davide Vercellino ${ }^{2}$ and Francesco Chiesa ${ }^{1}$
}

\begin{abstract}
Background: Cattle are intermediate hosts of six Sarcocystis species, among which Sarcocystis hominis and Sarcocystis heydorni can infect humans through the consumption of raw or undercooked meat. In addition to the zoonotic potential, there is increasing interest in these protozoa because of the evidence supporting the role of Sarcocystis spp. in the occurrence of bovine eosinophilic myositis (BEM), a specific inflammatory myopathy which leads to carcass condemnation and considerable economic losses. Actually, all the prevalence studies carried out on cattle in Italy have been based on either morphological or $18 \mathrm{~S}$ rDNA-based molecular techniques, most likely leading to misidentification of closely related species. Therefore, there is a strong need for new data on the prevalence of the different Sarcocystis spp. in cattle in Italy and their association with bovine eosinophilic myositis.

Methods: To reach our aim, individual striated muscle samples from BEM condemned carcasses $(N=54)$ and diaphragm muscle samples from randomly sampled carcasses $(N=59)$ were obtained from Northwest Italy slaughterhouses. Genomic DNA was extracted and analyzed by multiplex-PCR targeting 18S rDNA and cox 1 genes. PCR products amplified using the genus-specific primer set in absence of the specific fragment for S. hirsuta, S. cruzi, S. hominis or S. bovifelis were sequenced to achieve species identification.

Results: Sarcocystis DNA was detected in $67.8 \%$ of the samples from slaughter cattle and in $90.7 \%$ of the samples from BEM condemned carcasses. S. cruzi was identified as the most prevalent species in slaughter cattle (61\%), followed by S. bovifelis (10.2\%), S. hominis (8.5\%) and S. hirsuta (1.7\%). Notably, among the different Sarcocystis spp. detected, the presence of $S$. bovifelis and S. hominis was significantly higher in samples isolated from BEM condemned carcasses ( $46.3 \%$ and $40.7 \%$ respectively), while there was no statistically significant difference between the presence of S. cruzi or S. hirsuta in BEM condemned carcasses ( $42.6 \%$ and $1.8 \%$, respectively) and randomly sampled carcasses. Furthermore, DNA sequence analysis revealed the presence of a putative new species in two carcasses.

Conclusions: Our study contributes to updating the data on the prevalence of the different Sarcocystis spp. in cattle in Italy, highlighting the presence of three Sarcocystis spp., S. cruzi, S. hominis and S. bovifelis, in BEM lesions and allowing us to speculate on the possible role of $S$. hominis and $S$. bovifelis as the major sarcosporidian species involved in bovine eosinophilic myositis.
\end{abstract}

Keywords: Sarcocystis spp., Bovine eosinophilic myositis (BEM), Cox1 (COI) gene, 185 rDNA gene, Cattle, Molecular identification, Sarcocystis hominis, Zoonotic

\footnotetext{
*Correspondence: selene.rubiola@unito.it

1 Department of Veterinary Sciences, University of Turin, Largo Paolo

Braccini 2, 10095 Grugliasco, TO, Italy

Full list of author information is available at the end of the article
}

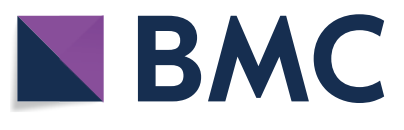

(c) The Author(s) 2021. This article is licensed under a Creative Commons Attribution 4.0 International License, which permits use, sharing, adaptation, distribution and reproduction in any medium or format, as long as you give appropriate credit to the original author(s) and the source, provide a link to the Creative Commons licence, and indicate if changes were made. The images or other third party material in this article are included in the article's Creative Commons licence, unless indicated otherwise in a credit line to the material. If material is not included in the article's Creative Commons licence and your intended use is not permitted by statutory regulation or exceeds the permitted use, you will need to obtain permission directly from the copyright holder. To view a copy of this licence, visit http://creativeco mmons.org/licenses/by/4.0/. The Creative Commons Public Domain Dedication waiver (http://creativecommons.org/publicdomain/ zero/1.0/) applies to the data made available in this article, unless otherwise stated in a credit line to the data. 


\section{Background}

Sarcocystis species are protozoan parasites belonging to the phylum Apicomplexa. The genus Sarcocystis consists of more than 200 species characterized by a worldwide distribution, three of which-S. hominis, S. heydorni and S. suihominis-are known to use humans as definitive hosts [1]. These latter become infected via the ingestion of cysts in muscular tissues, while the intermediate hosts acquire infection by ingesting oocysts and sporocysts in feed or water. Humans can develop two different clinical forms of sarcocystosis: an intestinal form, caused by $S$. hominis, S. heydorni and S. suihominis, and a muscular form, caused by $S$. nesbitti, the only Sarcocystis sp. that uses humans as intermediate hosts [1].

Among meat-producing animals, cattle (Bos taurus) are common intermediate hosts of Sarcocystis spp., whose prevalence in muscle can reach up to $100 \%$ [2]. Although there has recently been confusion about the validity and classification of several Sarcocystis spp. from cattle, it is now generally agreed that bovine muscle tissue can harbor at least six Sarcocystis spp., the well-known S. cruzi, S. hirsuta and S. hominis, with felids, canids and humans, respectively, as definitive hosts, and the recently added $S$. bovifelis, S. bovini and S. heydorni, with felids acting as definitive hosts for the first two species and primates acting as definitive hosts for the latter species [3-5].

The consumption of raw or undercooked beef meat constitutes an important risk factor for humans, who become infected by ingesting muscular sarcocysts [6]. Symptoms of intestinal sarcocystosis, such as nausea, abdominal pain and diarrhea, can have a wide range of intensity, depending on the number of ingested cysts and on the immune response of the host, though most infections go unnoticed [6]. In addition to the zoonotic potential, there is increasing interest around these protozoa in the food industry due to the evidence of their association with bovine eosinophilic myositis (BEM), a specific inflammatory myopathy with multifocal gray-green lesions which leads to carcass condemnation and considerable economic losses [7]. Worldwide, BEM reported prevalence in slaughtered cattle ranges from 0.002 to $5 \%$ [8]. These data might appear inconsistent with the high prevalence of Sarcocystis in cattle; in this regard, little evidence has pointed out as possible explanation that BEM might be associated with one or more Sarcocystis species [8].

Prevalence data reported from cattle in Italy are consistent with European reports, revealing a Sarcocystis spp. prevalence of $96 \%$ [9], 80\% [10], 91\% [11] and 88\% [12]. All these studies have been based on morphological techniques or on molecular techniques targeting the nuclear small subunit (18S) rDNA gene; however, the suitability of this locus for distinguishing between closely related Sarcocystis spp. has recently been challenged [13]. Indeed, though public databases contain mostly $18 \mathrm{~S}$ rDNA sequences because of its high use for Sarcocystis identification, cytochrome $\mathrm{C}$ oxidase subunit I mitochondrial (mtDNA cox 1 ) gene is actually seen as the most promising tool to differentiate closely related Sarcocystis spp. using ungulates as intermediate hosts $[3,14]$. In particular, as highlighted by Moré et al. [15], sequence differences among S. hominis, S. bovifelis and S. bovini are approximately $3 \%$ of the $18 \mathrm{~S}$ rDNA gene; therefore, using only size differences of the amplified $18 \mathrm{~S}$ rDNA fragments may result in the misidentification of these species $[2,15]$.

In the light of this, data resulting from the prevalence studies carried out in Italy in the last years might have led to an overestimation of $S$. hominis prevalence [16], apparently ranging from $42.7 \%$ [10] to $68 \%$ [12].

Thus, the aim of the present study was to evaluate the prevalence of the different Sarcocystis spp. in slaughter cattle from Northwest Italy and in BEM condemned carcasses, focusing on the hypothesis that BEM might be associated with specific Sarcocystis spp. [8, 11].

\section{Materials and methods}

\section{Sample collection and processing}

From January 2012 to July 2020, striated muscle samples from 54 BEM condemned carcasses were submitted by different slaughterhouses located in Northwest Italy to the Laboratory of Food Inspection at the Department of Veterinary Sciences (University of Turin, IT) for etiological confirmation. Muscle samples were macroscopically examined for the presence of typical focal or diffuse graygreen lesions (Fig. 1); detected lesions were excised and stored at $-20{ }^{\circ} \mathrm{C}$ for further analysis. Simultaneously, in 2019-2020 the diaphragm muscles of 59 slaughter cattle were collected from Piedmont (Northwest Italy) slaughterhouses, for a total of 113 individual cattle samples. Tissue samples were collected by veterinarians during post-mortem inspections of slaughtered animals and then transported to the laboratory at refrigeration temperature; $25 \mathrm{mg}$ of tissue for each individual muscle sample was collected and stored at $-20{ }^{\circ} \mathrm{C}$ until further analysis.

\section{DNA extraction and molecular detection of Sarcocystis spp.} DNA extraction was performed using DNeasy Blood and Tissue Kit (Qiagen, Hilden, Germany), according to the manufacturer's tissue protocol; the lysis step was carried out at $56{ }^{\circ} \mathrm{C}$ overnight with Proteinase K. DNA samples were eluted in $50 \mu \mathrm{l}$ of elution buffer and kept frozen at $-20{ }^{\circ} \mathrm{C}$. The identification of different Sarcocystis spp. was performed through the application of the multiplexPCR assay described by Rubiola et al. [16] targeting the 


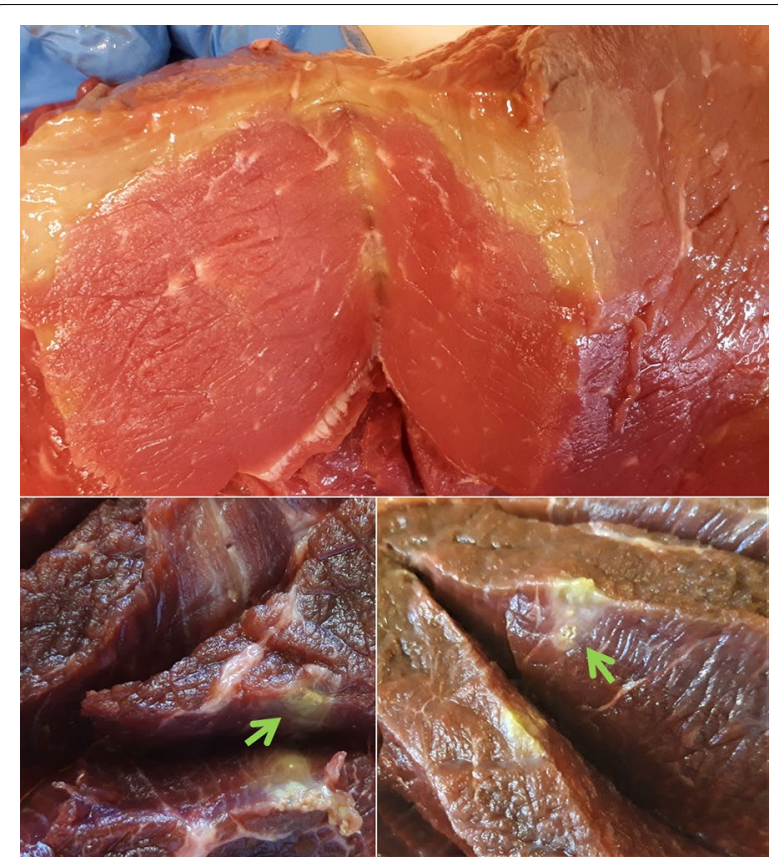

Fig. 1 Muscle samples with cut sections showing the presence of typical focal and diffuse gray-green lesions; central cores of suppuration are marked by the green arrows

$18 \mathrm{~S}$ rDNA gene and the mtDNA cox 1 gene. The multiplex-PCR contained $2.5 \mu \mathrm{l}$ of template DNA (5-20 ng/ $\mu \mathrm{l}), 0.5 \mathrm{mM}$ of each primer, Sarco Rev, Sar F, Hirsuta, Cruzi, COI HB, COI H and COI B, $2 \mathrm{mM} \mathrm{MgCl} 2,0.2 \mathrm{mM}$ of each dNTP, 1 U Platinum Taq DNA polymerase, $10 \times$ PCR buffer and RNase-free water to a total volume of $25 \mu$ l. The amplification was performed in an Applied Biosystems 2720 Thermal Cycler (AppliedBiosystems, CA, USA) with the following cycling profile: a denaturation step at $95^{\circ} \mathrm{C}$ for $3 \mathrm{~min}$, followed by 35 cycles at $95^{\circ} \mathrm{C}$ for $60 \mathrm{~s}, 58^{\circ} \mathrm{C}$ for $60 \mathrm{~s}$ and $72{ }^{\circ} \mathrm{C}$ for $30 \mathrm{~s}$ and final extension $72{ }^{\circ} \mathrm{C}$ for $3 \mathrm{~min}$. In each PCR run, $2.5 \mu \mathrm{l}$ of DNA from a collection of Sarcocystis-positive samples isolated from cattle striated muscle in the Department of Veterinary Science of Turin University [11, 16, 17] were used as positive controls while extracted DNA from negative cattle muscles as well as reagent blanks were included as negative controls. PCR products were observed in $2 \%$ agarose gel stained with SYBR safe stain (Invitrogen, Carlsbad, CA) and observed in a blue light transilluminator (Invitrogen, Groningen, The Netherlands).

\section{Sanger sequencing and phylogenetic analysis}

PCR products amplified through the use of the genusspecific primer set in the absence of the specific fragment for S. hirsuta, S. cruzi, S. hominis or S. bovifelis were sequenced to achieve species identification. PCR amplicons were purified with Exo-Sap treatment (USB Europe, Staufen, Germany) according to the manufacturer's instructions. Forward and reverse sequencing reactions were performed using ABI Prism BigDye Terminator Cycle Sequencing Ready Reaction Kit, version 1.1 (Applied Biosystems, Foster City, CA). Sequenced fragments were purified by DyeEX (Qiagen, Hilden, Germany), and sequence analysis was performed on an Applied Biosystems 310 Genetic Analyzer (Applied Biosystems, Foster City, CA). The nucleotide sequences were analyzed using the BLASTN sequence similarity search at the NCBI database [18]. Phylogenetic analyses of the $18 \mathrm{~S}$ rDNA gene sequences were performed using the neighbor-joining method [19] within MEGA7 [20]. Sarcocystis spp. reference sequences included are shown in Additional file 1.

\section{Statistical analysis}

Fisher's exact test was used to compare the proportions and 95\% confidence intervals (CIs) of different Sarcocystis spp. in BEM condemned carcasses and in slaughter cattle carcasses. $P \leq 0.05$ was considered significant.

\section{Results}

\section{Prevalence of Sarcocystis spp. in cattle carcasses}

Out of 59 individual samples from randomly sampled cattle carcasses, Sarcocystis DNA was detected in $67.8 \%$ (40/59; 95\% CI 55.06-78.36\%) of the muscle samples. S. cruzi was the most common species $(61 \%, 36 / 59 ; 95 \%$ CI 48.25-72.44\%), while S. bovifelis, S. hominis, S. hirsuta and an unidentified Sarcocystis sp. counted for 10.2\% (6/59; 95\% CI 4.40-20.81\%), 8.5\% (5/59; 95\% CI $3.27-18.75 \%), \quad 1.7 \%(1 / 59 ; 95 \% \quad \mathrm{CI}<0.01-9.85 \%)$ and 3.4\% (2/59; 95\% CI 0.26-12.22\%), respectively (Fig. 2a). Mixed infections were observed in $16.9 \%(n=10)$ of the samples, revealing the presence of up to two species of Sarcocystis at once. In cases of a single species being detected $(n=30), S$. cruzi was the most common finding $(86.7 \%, n=26)$, followed by S. bovifelis $(6.7 \%, n=2)$, S. hirsuta $(3.3 \%, n=1)$ and an unidentified Sarcocystis sp. $(3.3 \%, n=1)$. When two Sarcocystis spp. were detected in the same sample $(n=10)$, the simultaneous presence of $S$. cruzi and S. hominis was the most common finding (50\%), followed by co-infection of S. cruzi and S. bovifelis (40\%) and of S. cruzi and the unidentified Sarcocystis sp. (10\%), while no muscle samples revealed the simultaneous presence of three or more Sarcocystis spp.

Prevalence of Sarcocystis spp. in BEM condemned carcasses Out of 54 individual samples from BEM condemned carcasses, Sarcocystis DNA was detected in 90.7\% (49/54; 95\% CI: 79.67-96.40\%) of the muscle samples. The majority of intralesional Sarcocystis spp. were 

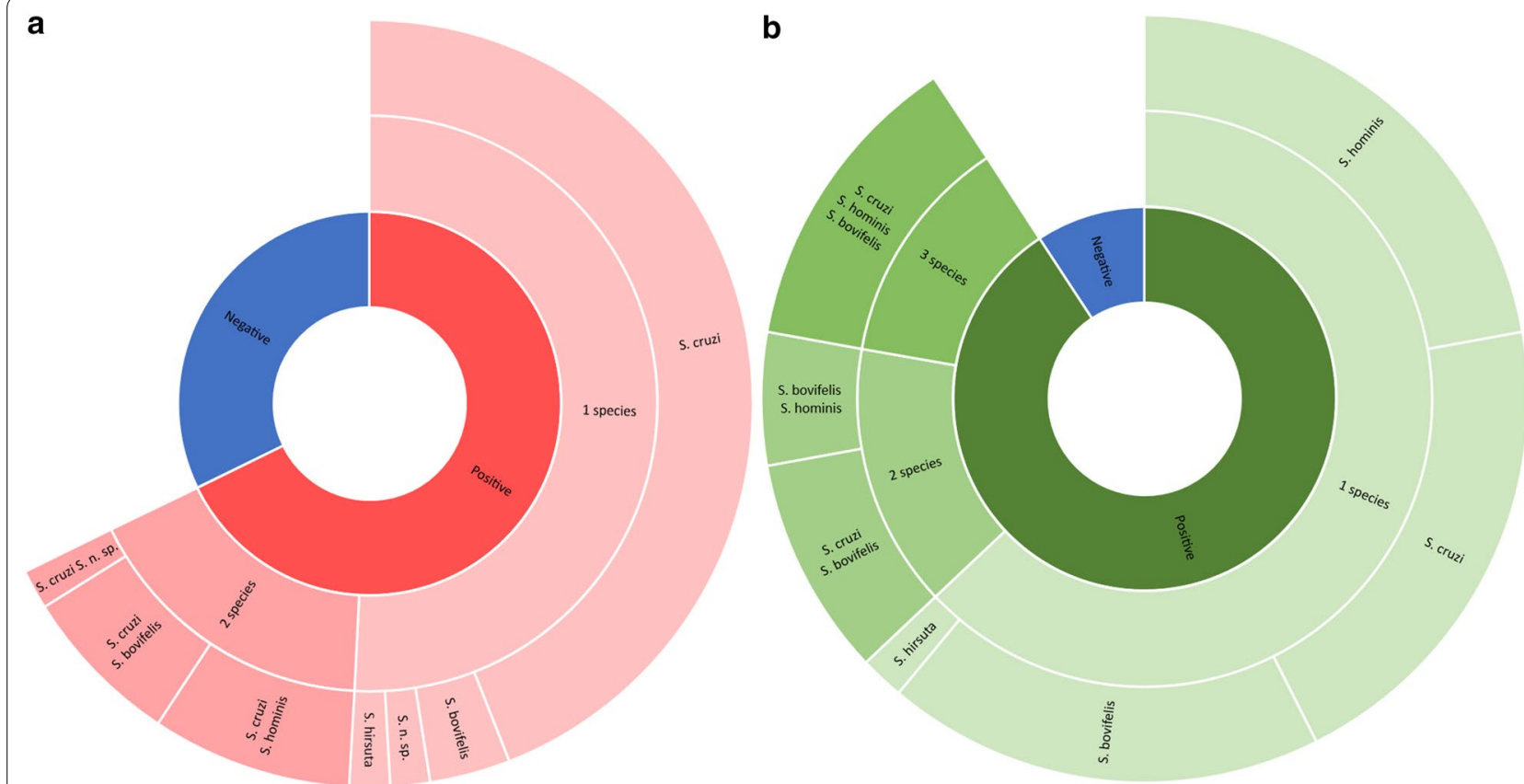

Fig. 2 Sunburst charts showing the distribution of different Sarcocystis spp. and co-infestations in slaughter cattle carcasses (a) and BEM condemned carcasses (b) analyzed in this study

found to be S. bovifelis $(46.3 \%, 25 / 54$, 95\% CI 33.69$59.40 \%)$, followed by S. cruzi $(42.6 \%, 23 / 54 ; 95 \%$ CI $30.32-55.85 \%)$ and S. hominis $(40.7 \%, 22 / 54,95 \%$ CI 28.66-54.05\%), while S. hirsuta counted for $1.8 \%$ (2/54; 95\% CI 0.30-13.26\%) (Fig. 2b). Mixed infections were observed in $27.8 \%(n=15)$ of the samples, revealing the presence of up to three species of Sarcocystis at once. Among co-infestations, the most common finding was the presence of two species $(53.3 \%, n=8)$, while three species were detected in seven samples (46.7\%). In case of a single species being detected $(n=34)$, S. hominis was the most common finding $(35.3 \%, n=12)$, followed by $S$. cruzi $(32.4 \%, n=11), S$. bovifelis $(29.4 \%, n=10)$ and S. hirsuta $(2.9 \%, n=1)$. When two Sarcocystis spp. were detected in the same sample, the simultaneous presence of $S$. cruzi and S. bovifelis was the most common finding (62.5\%), followed by co-infection of $S$. bovifelis and S. hominis (37.5\%), while all muscle samples harboring three species revealed the simultaneous presence of S. cruzi, S. bovifelis and S. hominis.

\section{Sanger sequencing and phylogenetic analysis}

Two out of 59 individual samples from randomly sampled cattle carcasses revealed the presence of a PCR product amplified through the use of the genus specific primer set, in the absence of the specific fragment for S. hirsuta, S. cruzi, S. hominis or S. bovifelis. Consensus sequences of the $18 \mathrm{~S}$ rDNA fragments were $152 \mathrm{bp}$ in length and showed $<86.43 \%$ similarity to any known Sarcocystis spp. sequence deposited in GenBank. Notably, these amplicons showed a sequence homology $\geq 95.42-99.34 \%$ with three GenBank entries (accession no. FN394498-FN394500) corresponding to unidentified Sarcocystis spp. isolated from cattle muscle samples [8]. The obtained 18S rDNA sequences were deposited in Genbank under accession no. MW582306, MW582307. A phylogenetic analysis on the unidentified 18S rDNA sequences and on representative sequences deposited in GenBank was inferred using the neighbor-joining method [19] within MEGA7 [20]; the resulting phylogenetic tree is shown in Fig. 3. The unidentified 18S rDNA sequences from this study formed a monophyletic cluster together with GenBank entries

\footnotetext{
(See figure on next page.)

Fig. 3 Neighbor-joining phylogenetic tree for members of the Sarcocystidae based on 185 rDNA sequences of 37 Sarcocystis spp. and including the unidentified Sarcocystis spp. sequences isolated in this study (in bold) and three GenBank entries (Accession Nos. FN394500.1, FN394498.1, FN394499.1) corresponding to unidentified Sarcocystis spp. [8]. Toxoplasma gondii and Neospora caninum were used as outgroups. The percentage of replicate trees in which the associated taxa clustered together in the bootstrap test (1000 replicates) are shown next to the branches. Bootstrap values $<50$ are not shown
} 


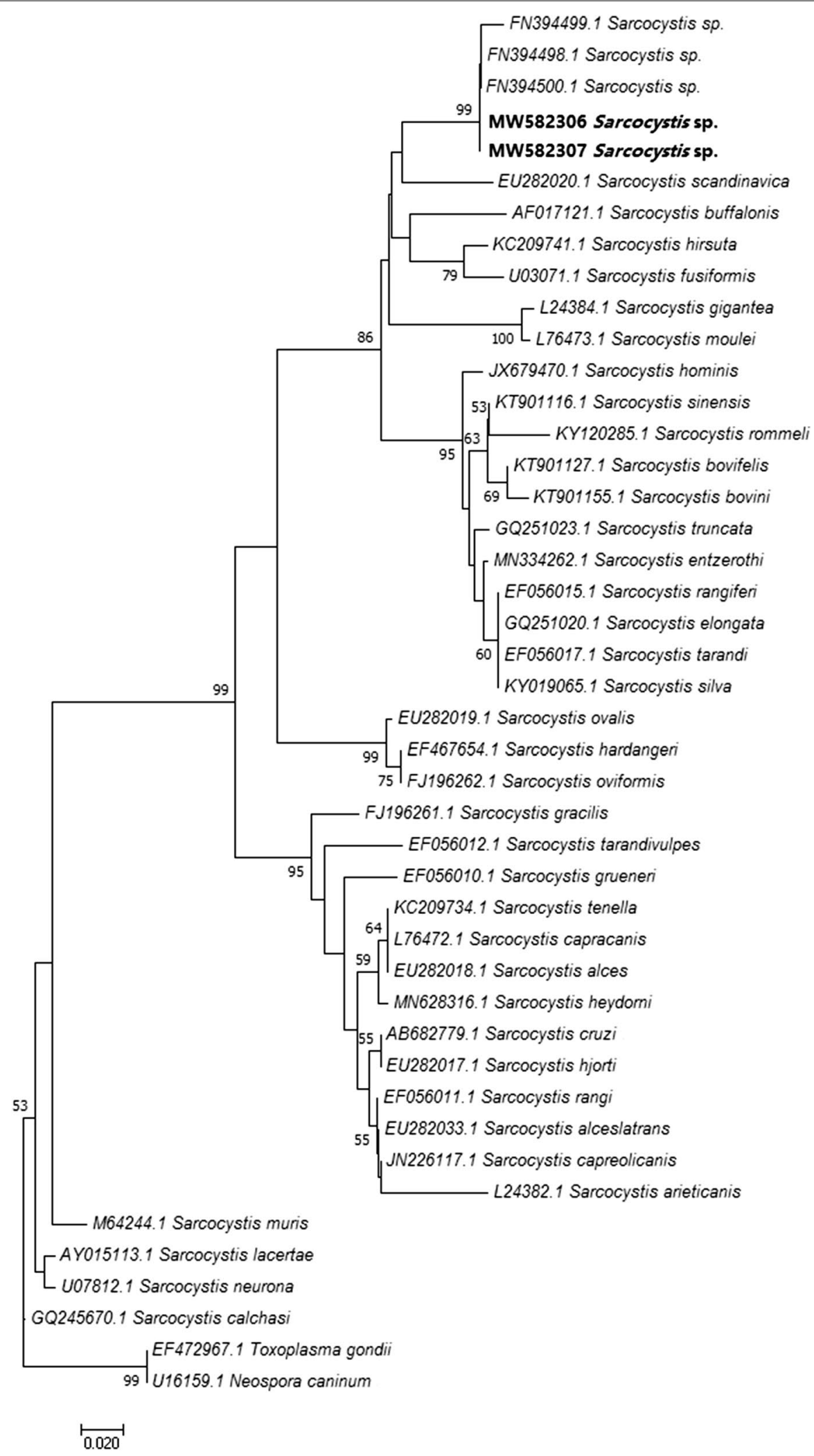


FN394498-FN394500 within the clade including cattransmitted Sarcocystis spp. with ruminant intermediate hosts.

\section{Statistical analysis}

The presence of Sarcocystis spp. DNA was significantly higher in samples isolated from BEM condemned carcasses than in samples isolated from randomly sampled cattle carcasses (Fisher's exact test two-tailed, $P=0.0050$ ). Among the different Sarcocystis spp. detected, the presence of S. bovifelis or S. hominis was significantly higher in samples isolated from BEM condemned carcasses than in samples isolated from randomly sampled cattle carcasses (Fisher's exact test two-tailed, $P<0.0001$ ), while there was no statistically significant difference between the presence of S. cruzi, S. hirsuta or the unidentified Sarcocystis sp. in BEM condemned carcasses and randomly sampled carcasses (Fisher's exact test two-tailed, $P=0.0606, P>0,9999, P=0,4965$, respectively).

\section{Discussion}

Cattle sarcocystosis is gaining importance as one of the causes of bovine eosinophilic myositis, a specific inflammatory myopathy which leads to serious economic outcomes in the beef sector [2,11]. Thus, species identification of intra-lesional Sarcocystis is crucial to better understand the contribution of specific species to BEM pathogenesis and to explain the low prevalence of BEM lesions [16], despite the high prevalence of sarcocysts in the cattle population. Therefore, the aim of this study was to evaluate the presence of Sarcocystis spp. in Italian slaughter cattle and in BEM condemned carcasses to update the prevalence data reported from cattle in Italy in light of the recent taxonomic revision of cattle Sarcocystis and to evaluate the hypothesis that BEM might be associated with specific Sarcocystis spp. [8].

In our study, the $67.8 \%$ prevalence of Sarcocystis spp. in diaphragm samples randomly taken from Northwest Italy slaughterhouses $(n=59)$ is compatible with the high prevalence previously reported [9-12]. S. cruzi has been confirmed as the most common species, followed by $S$. bovifelis and S. hominis, while S. hirsuta DNA was only detected in $1.7 \%$ of samples. Notably, the $8.5 \%$ prevalence of $S$. hominis here detected is much lower than previously reported (43-68\%), while S. bovifelis has never been considered in prevalence studies carried out in Italy so far [9-12], since at that time it still had to be described [21]. These findings highlight the previous overestimation of $S$. hominis prevalence due to the detection techniques based on the lower discriminative $18 \mathrm{~S}$ rDNA gene; this evidence suggests that $S$. bovifelis might have been misidentified as $S$. hominis, thus explaining its absence in all previous prevalence studies carried out in Italy [16]. Furthermore, the examined striated and diaphragm muscle samples have proved to be suitable matrices to investigate the presence of different Sarcocystis spp. This tendency has been previously confirmed in several studies [10, 22, 23] examining different tissue (e.g. heart, skeletal muscle, esophagus, diaphragm, tongue) and confirmed by others processing only heart muscle samples $[24,25]$. Therefore, the tissue matrices can considerably influence the detection of different Sarcocystis spp.

Considering the high detection of S. cruzi, followed by S. bovifelis and S. hominis, the prevalence data reported in our study show most resemblance to those of Hungary [22] and The Netherlands [26], while in Germany and Lithuania a higher prevalence of S. hirsuta is reported, ranging from 6.6 to $30.4 \%$, respectively $[27,28]$.

The presence of Sarcocystis spp. in BEM condemned carcasses differed significantly with respect to the previously described group of randomly sampled slaughter cattle. In particular, the detection of Sarcocystis spp. DNA in $90.7 \%$ of the BEM condemned carcasses was significantly higher than in unaffected cattle. This finding confirms the association of Sarcocystis spp. with BEM lesions, though the presence of Sarcocystis spp. is not exclusively associated with lesions typical for bovine eosinophilic myositis [26]. Besides, among the different Sarcocystis spp. detected, the presence of S. bovifelis and $S$. hominis was significantly higher in samples isolated from BEM condemned carcasses than in samples isolated from randomly sampled slaughter cattle. This finding supports the hypothesis that BEM might be associated with specific Sarcocystis spp. Literature on this topic is confusing since several species have been reported in association with eosinophilic lesions [7, 8, 26, 29-32]; among these studies, both thin- and thickwalled Sarcocystis spp. are reported, including S. hominis [7, 8, 26, 29] and S. cruzi [32]. However, most of these reports have been based on morphological identification, which cannot discriminate among closely related Sarcocystis spp.; besides, this method is affected by the damage of the cyst walls which is often present in BEM lesions [8]. Molecular detection techniques were applied by Vangeel et al. [8], and the majority of intralesional Sarcocystis were found to be $S$. hominis, though also $S$. cruzi and $S$. hirsuta were found in BEM lesions; however, S. bovifelis was not recognized as a different Sarcocystis spp. until its resurrection in 2016 [21]. Our findings lead us to hypothesize a major role for $S$. hominis and S. bovifelis in bovine eosinophilic myositis, though also $S$. cruzi was detected in BEM condemned carcasses. To corroborate our current findings, as well as to update knowledge on this disease, which leads to serious economic outcomes in the beef sector, further 
research is required. In particular, multiple sampling in BEM-affected carcasses, involving both intralesional and extralesional tissue, might be performed to evaluate the presence of different Sarcocystis spp. outside and inside lesions.

In the present study, an unidentified species was detected in two carcasses; although the small size of the sequenced fragment cannot give consistent molecular results, as highlighted by the low bootstrap values reported in the phylogenetic tree (Fig. 3), this species seems to be most closely related to thick-walled Sarcocystis spp. from bovids and cervids. Interestingly, as suggested by the high percentage of identity reported in the phylogenetic analysis (Fig. 3), a 177-bp 18S rDNA fragment of this unidentified species has already been sequenced in association with BEM lesions in Belgium [8], though no further research was performed at that time. Further investigations are needed to characterize this putative new species and investigate its cycle and possible role in BEM pathogenesis.

The detection of the zoonotic S. hominis confirms the established transmission cycle between cattle and humans in Italy, pointing out the risk for the consumer of raw or undercooked beef. Human intestinal sarcocystosis is well documented in the literature, in both asymptomatic patients and patients with gastrointestinal symptoms [6]. Recently, the presence of S. hominis in six patients hospitalized with gastrointestinal symptoms has been reported in the Piedmont region, Northwest Italy, which is well known for raw beef consumption [16]. Therefore, epidemiological data on this and other actually undetected zoonotic species must be considered of importance from a public health perspective.

\section{Conclusions}

In conclusion, the results of our study contribute to updating the data on the prevalence of the different Sarcocystis spp. from cattle in Italy, highlighting the previous overestimation of $S$. hominis due to the use of morphological methods or ineffective $18 \mathrm{~S}$ rDNA-based molecular techniques. Besides, our findings contribute to the understanding of the importance of different Sarcocystis spp. in BEM pathogenesis, highlighting the presence of three species, S. cruzi, S. hominis and S. bovifelis, in BEM lesions and allowing us to speculate on the possible role of $S$. hominis and S. bovifelis as the major sarcosporidian species involved. Lastly, considering the detection of the zoonotic $S$. hominis, the results of the current survey highlight a substantial public health concern and offer useful information for public health specialists.

\section{Supplementary Information}

The online version contains supplementary material available at https://doi. org/10.1186/s13071-021-04722-5.

Additional file 1: Table S1. Reference sequences downloaded from GenBank and used in this study.

\section{Acknowledgements}

We are grateful to Professor Bjørn Kåre Gjerde (Norwegian University of Life Sciences-NMBU) for fruitful discussions and support.

\section{Authors' contributions}

SR performed the experiments and the data analysis and wrote the manuscript. TC an FC conceived and designed the experiments. FP assisted the experiments. DV carried out the sampling. All authors read and approved the final manuscript.

\section{Funding}

Not applicable: this research did not receive any specific grant from funding agencies in the public, commercial, or not-for-profit sectors.

\section{Availability of data and materials}

All datasets generated for this study are included in article/additional files. The sequences generated in the present study are available in GenBank database with Accession Numbers MW582306, MW582307.

\section{Declarations}

Ethics approval and consent to participate

Not applicable: for this type of formal study consent is not required.

Consent for publication

Not applicable.

\section{Competing interests}

The authors declare that the research was conducted in the absence of any commercial or financial relationships that could be construed as a potential conflict of interest.

\section{Author details}

${ }_{1}^{1}$ Department of Veterinary Sciences, University of Turin, Largo Paolo Braccini 2, 10095 Grugliasco, TO, Italy. ${ }^{2}$ Veterinary Service (A.S.L. AT), 14100 Asti, AT, Italy.

Received: 18 February 2021 Accepted: 7 April 2021

Published online: 23 April 2021

References

1. Dubey JP. Foodborne and waterborne zoonotic sarcocystosis. Food Waterborne Parasitol. 2015;1:2-11.

2. Vangeel L, Houf K, Chiers K, Vercruysse J, D’herde K, Ducatelle R. Molecular-based identification of Sarcocystis hominis in Belgian minced beef. J Food Prot. 2007:70:1523-6.

3. Gjerde B. Molecular characterisation of Sarcocystis bovifelis, Sarcocystis bovini n. sp., Sarcocystis hirsuta and Sarcocystis cruzi from cattle (Bos taurus) and Sarcocystis sinensis from water buffaloes (Bubalus bubalis). Parasitol Res. 2016;115:1473-92.

4. Hu JJ, Wen T, Chen XW, Liu TT, Esch GW, Huang S. Prevalance, morphology, and molecular characterization of Sarcocystis heydorni sarcocysts from cattle (Bos taurus) in China. J Parasitol. 2016;102:545-8.

5. Prakas P, Balčiauskas L, Juozaitytè-Ngugu E, Butkauskas D. The role of mustelids in the transmission of Sarcocystis spp. using cattle as intermediate hosts. Animals. 2021;11:822.

6. Fayer R, Esposito DH, Dubey JP. Human infections with Sarcocystis species. Clin Microbiol Rev. 2015:28:295-311.

7. Wouda W, Snoep JJ, Dubey JP. Eosinophilic myositis due to Sarcocystis hominis in a beef cow. J Comp Pathol. 2006;135:249-53. 
8. Vangeel L, Houf K, Geldhof P, de Preter K, Vercruysse J, Ducatelle R, et al. Different Sarcocystis spp. are present in bovine eosinophilic myositis. Vet Parasitol. 2013;197:543-8.

9. Bucca M, Brianti E, Giuffrida A, Ziino G, Cicciari S, Panebianco A. Prevalence and distribution of Sarcocystis spp. cysts in several muscles of cattle slaughtered in Sicily Southern Italy. Food Control. 2011;22:105-8.

10. Domenis L, Peletto S, Sacchi L, Clementi E, Genchi M, Felisari L, et al. Detection of a morphogenetically novel Sarcocystis hominis-like in the context of a prevalence study in semi-intensively bred cattle in Italy. Parasitol Res. 2011;109:1677-87.

11. Chiesa F, Muratore E, Dalmasso A, Civera T. A new molecular approach to assess the occurrence of Sarcocystis spp. in cattle and products thereof: preliminary data. Ital J Food Saf. 2013;2:41.

12. Meistro S, Peletto S, Pezzolato M, Varello K, Botta M, Richelmi G, et al. Sarcocystis spp. prevalence in bovine minced meat: a histological and molecular study. Ital J Food Saf. 2015;4(2):4626.

13. Robertson LJ, Clark CG, Debenham JJ, Dubey JP, Kváč M, Li J, et al. Are molecular tools clarifying or confusing our understanding of the public health threat from zoonotic enteric protozoa in wildlife? Int J Parasitol Parasites Wildl. 2019;9:323-41.

14. Gjerde B. Phylogenetic relationships among Sarcocystis species in cervids, cattle and sheep inferred from the mitochondrial cytochrome c oxidase subunit I gene. Int J Parasitol. 2013;43:579-91.

15. Moré G, Schares S, Maksimov A, Conraths FJ, Venturini MC, Schares G. Development of a multiplex real time PCR to differentiate Sarcocystis spp. affecting cattle. Vet Parasitol. 2013;197:85-94.

16. Rubiola S, Civera T, Ferroglio E, Zanet S, Zaccaria T, Brossa S, et al. Molecular differentiation of cattle Sarcocystis spp. by multiplex PCR targeting $18 \mathrm{~S}$ and $\mathrm{CO}$ genes following identification of Sarcocystis hominis in human stool samples. Food Waterborne Parasitol. 2020;18:e00074.

17. Rubiola S, Chiesa F, Zanet S, Civera T. Molecular identification of Sarcocystis spp. in cattle: partial sequencing of Cytochrome C Oxidase subunit 1 (COI). Ital J Food Saf. 2018;7(4):7725.

18. Altschul SF, Gish W, Miller W, Myers EW, Lipman DJ. Basic local alignment search tool. J Mol Biol. 1990;215:403-10.

19. Saitou N, Nei M. The neighbor-joining method: a new method for reconstructing phylogenetic trees. Mol Biol Evol. 1987;4:406-25.

20. Kumar S, Stecher G, Tamura K. MEGA7: Molecular Evolutionary Genetics Analysis version 7.0 for bigger datasets. Mol Biol Evol. 2016;33:1870-4.

21. Gjerde B, The resurrection of a species: Sarcocystis bovifelis Heydorn, , et al. is distinct from the current Sarcocystis hirsuta in cattle and morphologically indistinguishable from Sarcocystis sinensis in water buffaloes. Parasitol Res. 1975;2016(115):1-21.

22. Hornok S, Mester A, Takács N, Baska F, Majoros G, Fok É, et al. Sarcocystisinfection of cattle in Hungary. Parasites Vectors. 2015;8:69.

23. Yang $Y$, Dong $H$, Su $R$, Wang $Y$, Wang $R$, Jiang $Y$, et al. High prevalence of Sarcocystis spp. infections in cattle (Bos taurus) from central China. Parasitol Int. 2018;67:800-4.

24. Imre K, Dărăbuș G, Tîrziu E, Morariu S, Imre M, Plutzer J, et al. Sarcocystis spp. in romanian slaughtered cattle: molecular characterization and epidemiological significance of the findings. Biomed Res Int. 2019;2019:4123154

25. Ferreira MST, Vogel FSF, Sangioni LA, Cezar AS, Braunig P, de Avilla BS, et al. Sarcocystis species identification in cattle hearts destined to human consumption in southern Brazil. Vet Parasitol Reg Stud Rep. 2018;14:94-8.

26. Hoeve-Bakker BJA, van der Giessen JWB, Franssen FFJ. Molecular identification targeting cox 1 and $18 \mathrm{~S}$ genes confirms the high prevalence of Sarcocystis spp in cattle in the Netherlands. Int J Parasitol. 2019;49:859-66.

27. Moré G, Pantchev A, Skuballa J, Langenmayer MC, Maksimov P, Conraths FJ, et al. Sarcocystis sinensisis the most prevalent thick-walled Sarcocystis species in beef on sale for consumers in Germany. Parasitol Res. 2014;113:2223-30.

28. Prakas P, Strazdaitè-Žielienè Ž, Januškevičius V, Chiesa F, Baranauskaitè A, Rudaitytè-Lukošienè E, et al. Molecular identification of four Sarcocystis species in cattle from Lithuania, including S. hominis, and development of a rapid molecular detection method. Parasites Vectors. 2020;13:610.

29. Rimaila-Pärnänen E, Nikander S. Generalized eosinophilic myositis with sarcosporidiosis in a Finnish cow. Nord Vet Med. 1980;32:96-9.

30. Jensen R, Alexander AF, Dahlgren RR, Jolley WR, Marquardt WC, Flack DE, et al. Eosinophilic myositis and muscular sarcocystosis in the carcasses of slaughtered cattle and lambs. Am J Vet Res. 1986;47:587-93.

31. Gajadhar AA, Yates WD, Allen JR. Association of eosinophilic myositis with an unusual species of Sarcocystis in a beef cow. Can J Vet Res. 1987:51:373-8.

32. Gajadhar AA, Marquardt WC. Ultrastructural and transmission evidence of Sarcocystis cruzi associated with eosinophilic myositis in cattle. Can J Vet Res. 1992:56:41-6.

\section{Publisher's Note}

Springer Nature remains neutral with regard to jurisdictional claims in published maps and institutional affiliations.
Ready to submit your research? Choose BMC and benefit from:

- fast, convenient online submission

- thorough peer review by experienced researchers in your field

- rapid publication on acceptance

- support for research data, including large and complex data types

- gold Open Access which fosters wider collaboration and increased citations

- maximum visibility for your research: over 100M website views per year

At BMC, research is always in progress.

Learn more biomedcentral.com/submissions 